\title{
The Stiffness of Syntactic Metal-Matrix Composites: A Statistical Model
}

\author{
J. D. Botas ${ }^{1}$ and H. Águas ${ }^{2}$ \\ ${ }^{1}$ Materials Science Department (DCM), Faculty of Science and Technology, New University of Lisbon, \\ Quinta da Torre, 2829-516 Caparica, Portugal \\ ${ }^{2}$ CENIMAT/I3N, Departamento de Ciência dos Materiais, Faculdade de Ciências e Tecnologia (FCT), \\ Universidade Nova de Lisboa, 2829-516 Caparica, Portugal
}

Correspondence should be addressed to H. Águas, hma@fct.unl.pt

Received 29 September 2011; Accepted 19 October 2011

Academic Editor: Y. Waku

Copyright ( $\odot 2011$ J. D. Botas and H. Águas. This is an open access article distributed under the Creative Commons Attribution License, which permits unrestricted use, distribution, and reproduction in any medium, provided the original work is properly cited.

Stiffness estimates of unloaded isotropic particulates are made by a new analytical model, when reinforcements are either compact or hollow spheres. A statistical extension of this model is described when stiffness predictions involve loading of syntactic composites. A simple experimental routine is also proposed for monitoring the microballoons fracture upon brittle syntactic metal-matrix composites tensile loading.

\section{Introduction}

Composite materials have managed to stand in the forefront of engineering materials, mainly because of their structural abilities. Among composite materials rank the particle composites ("particulates") as the cheapest of them all. The spherical particulates emergence has been dictated by the spherical reinforcements surface ability to avoid stress concentrations, thus minimizing the cracking likelihood of the surrounding matrices. More recently, the replacement of compact spherical reinforcements by hollow ones ("balloons") led to a novel class of foam-like materials, the socalled "syntactic composites." The hollow spheres homogeneous morphology as well as their distribution modes once embedded in various matrices, impart to the overall composite structure some relevant properties-for example, low density, good energy absorption, and enhanced fracture toughness over their compact reinforcements composite counterparts. Among other current techniques, dispersions of ceramic balloons in metal matrices may be achieved by infiltrating molten metal around a preform of uniform hollow spheres, whose packing modes may eventually range from a random distribution to an ordered pattern. In accordance with the balloons extreme packing modes, a random balloons distribution will imply a fully isotropic syntactic composite whereas an hexagonal close-packed array will determine an extreme anisotropic composite behaviour. In practice, an anisotropy-free composite can be obtained as long as its balloon concentration does not surpass a certain upper bound value. Such condition ensures no significant balloon clusters formation, thus preventing "crystal-like patterns" to occur within the composite. This preventive measure implies to disqualify for design purposes any empirical formula or approach where the concentration restriction may not be accounted for (as in the conventional rule of mixtures).

Experimental data on syntactic composites [1] currently span from basic mechanical to other nonstructural properties. The former case may include elastic stiffness, specific tensile/compressive strength, and static fracture toughness, whereas the latter may refer to dielectric behaviour, thermal conductivity, and absorption of acoustic noise/resonance. Some of the experimental results pertaining to the above topics may anyhow be affected by poor reliability as they were treated as isotropic despite being investigated at high volume fraction ranges or assessed by simulation or testing methods in disregard of the materials surface flaws. These shortcomings are further aggravated by the notorious lack 
of widespread modelling of syntactic composites properties, which often leaves experimental research without reference predictions from a theoretical framework. So, in order to overcome some of the above drawbacks, this work will resort to micromechanical concepts to produce elastic stiffness estimates for an ensemble of uniformly sized elastic balloons randomly embedded into an isotropic elastic matrix. Interfaces between matrix and balloons shall not be accounted for by this model, which therefore suits actual composites whose processing involves only moderate temperatures and/or sluggish interdiffusion rates (i.e., balloon reinforced composites, whose interfaces thickness is either small or negligible).

In this form, the present work derives from an early micromechanics model [2] and is the end result of some publications devoted to the stiffness of spherical particulates. The basic model, hereafter termed Paul's model, was selected for its sensitiveness towards reinforcement morphologies and simple formulation to achieve the Young modulus of a particulate. This early model was subsequently reformulated so as to predict the stiffness of solid sphere particulates [3] as well as that of hollow sphere particulates [4].

The modified model also enables density forecasts for either compact or hollow sphere particulates, and its capability was extended so as to predict the unsteady stiffness of fragile hollow sphere particulates when subjected to uniaxial tensile loading. Since the ceramic balloon composites and the balloons themselves can be regarded as brittle solids, their failure at a particular applied tensile stress depends on the statistical existence of surface flaws or cracks. The proposed model for the stiffness of loaded syntactic composites is therefore a statistical model. Gathering mechanical information adequate enough for predicting the elastic stiffness of an isotropic solid is just a part of a broader theoretical frame aiming at determining the two independent elastic constants which define that solid elastic behaviour. As regards the isotropic composites, that search requires a statistically isotropic (random) distribution of their (also elastic isotropic) constitutive reinforcements-which are assumed in the present analysis to be an ensemble of uniform spheres, embedded in a continuous and isotropic elastic matrix. Some current advanced approaches aiming at computing the isotropic composite independent constants span from analytical models [5-9] to numerical methods $[10,11]$, which are to be briefly described next. A more detailed account of these models can be found in the last section of this paper, where conclusions are drawn.

The earliest of the above referred analytical models is the Mori-Tanaka mean-field approximation [5], later applied to isotropic composites by Benveniste [6] by using the same principles yet accounting for different elastic interactions between the inclusions. Analytical expressions are provided by these models from which the independent elastic constants can be obtained. An alternative analytical model was later developed by Christensen and Lo [7], the socalled generalized self-consistent approach, where the elastic constants are obtained by solving some differential equations under appropriate boundary conditions. A more recent analysis is the Torquato's third-order approximation $[8,9]$, where the independent constants can be derived from two statistical correlation parameters, plus the first three terms of a truncated series of the effective stiffness tensor serial expansion. As for the numerical "exact" solution, the required two independent elastic constants are usually computed by the finite element method as applied to a representative volume element (commonly, a cubic cell) under periodic boundary conditions. Such a volume element contains a random dispersion of (spherical) isotropic reinforcements so as all directions within the unit cell to be statistically equivalent; a requirement made easier to comply with once Drugan and Willis [10] and Drugan [11] demonstrated that an arrangement of some dozen spheres was good enough to secure the volume element statistical representativeness.

\section{The Paul Model}

This model assumes that the states of macroscopic stress and strain imposed on a particulate by an external tensile stress can be reproduced in a typical unit volume, which consists of a single particle embedded in a unit cube of matrix. Additional approximations regarding the particulate constituents further assume both matrix and particles are subjected to the same strain and have the same Poisson's ratios - a mechanical condition which best suits metal/ceramic combinations. Adhesion is also assumed to be maintained at the particle/matrix interface, when the unit cube of matrix becomes strained by an internal tensile force along the $x$ direction.

The above conditions yield the elastic modulus $E$ of the composite material

$$
\begin{aligned}
\frac{1}{E} & =\int_{0}^{1} \frac{d x}{E_{m}+\left(E_{p}-E_{m}\right) \cdot A(x)} \\
& \equiv \int_{0}^{1} \frac{d x}{E_{p}+\left(E_{m}-E_{p}\right) \cdot B(x)},
\end{aligned}
$$

where $E_{m}$ and $E_{p}$ are the "matrix" and the "particle" moduli and $A(x)$ and $B(x)$ are the "particle..." and the "matrix" morphology distribution functions along the $x$ direction, which comply with the $\{A(x)+B(x)=1\}$ condition at any integration stage. The first equality is usually preferred because it is easier to describe a particle morphology within the unit volume element rather than that of the surrounding matrix.

In predicting just a single composite stiffness value regardless the stiffness values arising from other material directions, this early model implicitly acknowledges that the particulates under assessment are to be isotropic composites. From which follows that the above stiffness predictions are valid providing the composites reinforcement content lies in a safe concentration range, where nucleation of particle patterns does not occur significantly.

Equation (1) integration was carried out for a cubicshaped particle within the matrix cubic cell, yielding the cubic-particles composite stiffness $\left(E_{c}\right)$ for a cermet material

$$
E_{c}=E_{m}\left\{\frac{1+(\varphi-1) V_{p}^{2 / 3}}{1+(\varphi-1)\left(V_{p}^{2 / 3}-V_{p}\right)}\right\},
$$


where $V_{p}$ is the volume fraction of the cubic particle (of tungsten carbide) in the cermet, and $\varphi \equiv\left(E_{p} / E_{m}\right)$ is the modulus ratio of the particle to the matrix.

\section{The Modified Paul Model}

Paul's analysis approached an actual particles composite as if its overall properties matched those of a representative volume element. Such volume element was a cubic matrix cell enclosing a solid cubic-shaped virtual particle, whose size was adjusted by the actual particles volume fraction. This reasoning is maintained in the modified model $[3,4]$, which deals with random dispersions of minute ceramic spherical particles embedded in a metal matrix. Such spheres may either be compact ("microspheres") or hollow ("microballoons"). The "modified" model representative volume element differs from that of the original model in the spherical shape (rather than cubic) of the virtual particle enclosed by the cubic matrix unit cell. Moreover, the modified model enables a theoretical provision for a safe concentration range within which the spherical particles dispersion can remain isotropic (theoretical volume fraction upper bound $=0.52$ ).

When metal matrices become reinforced by ceramic microspheres, so that $\varphi \equiv\left(E_{s} / E_{m}\right)>1$, where $E_{s}$ is the microspheres Young modulus, this model predicts a composite stiffness $E_{S}$ as indicated below

$$
E_{S}=E_{m}\left\{(1-2 R)-\frac{1}{\beta \sqrt{R^{2}+1 / \beta}} \ln \left|\frac{R-\sqrt{R^{2}+1 / \beta}}{R+\sqrt{R^{2}+1 / \beta}}\right|\right\}^{-1},
$$

where $R=0.62 V_{s}^{1 / 3}$ is the virtual particle radius, $V_{s}$ is the microspheres volume fraction within the unit cell, and $\beta=$ $[\pi(\varphi-1)]$ is a material constant.

However, when metal matrices become reinforced by ceramic thin-walled microballoons so that $\varphi \equiv\left(E_{b} / E_{m}\right)<1$, where $E_{b}$ is the microballoons Young modulus, the elastic modulus $E_{B}$ of the syntactic composite is predicted by the modified model as follows:

$$
E_{B}=E_{m}\left\{(1-2 R)+\frac{2 c}{\sqrt{c-R^{2}}} \arctan \left[\frac{R}{\sqrt{c-R^{2}}}\right]\right\}^{-1},
$$

where $R=0.62 V_{b}^{1 / 3}, V_{b}$ is the microballoons volume fraction within the unit cell, $c=[\pi(1-\varphi)]^{-1}$ is a material constant, and the microballoons elastic modulus $E_{b}$ (as required by the constant $c$ evaluation) can be derived from a constitutive equation concerning low-density foams

$$
E_{b}=E_{\mathrm{mon}}\left[1-\left(\frac{d}{D}\right)^{3}\right]^{k} .
$$

In the above, $E_{\mathrm{mon}}$ is the Young modulus of the balloons monolithic material, $d$ and $D$ are the inner and outer diameters of the actual balloons, and $k$ is a "porosity factor" (=2 for open-cell foams; $=3$ for closed-cell foams; and between these values for quasi-closed cell foams) whose


(1) $E_{S}$ - Alumina/Al-alloy
(4) $E_{B} \longrightarrow$ Alumina/Al-alloy
(2) $E_{S} \longrightarrow$ Silceram/Al-alloy
(3) $E_{S} \longrightarrow$ Mild steel/Al-alloy
(5) $E_{B} \longrightarrow$ Silceram/Al-alloy
(6) $E_{B} \longrightarrow$ Mild steel/Al-alloy

Figure 1: The stiffness of spherical particulates, as predicted by (3) (compact particles) and (4) (hollow particles) of this model with $k=2.5$.

former value [4] was 2.5 but should rather be taken within the 2.7-2.9 range so as to approach closer some recent experimental data [12].

The fundamental equations of this modified model (i.e., (3), (4), (5)) account for the stiffness of particulate metalmatrix composites as long as the reinforcement volume fraction is not greater than $52 \%$ and remains constant throughout uniaxial tensile loading. If so, the particles dispersion remains isotropic, and the reinforcement volume fraction remains invariant because the particles population is not destroyed while the composites are subjected to external stresses. Providing these conditions are met, a "modified model" application can be illustrated by an example, where a single balloon morphology $(D=50 \mu \mathrm{m}, d=45 \mu \mathrm{m}$; thus shell thickness $=2.5 \mu \mathrm{m}$, and $E_{b}=3.82 \times 10^{-2} E_{\text {mon }}$ after (5) with a cell-porosity exponent $=2.5$ ) is utilized for three distinct microballoon materials embedded in the same light-(Al)alloy matrix $\left(E_{m}=71 \mathrm{GPa}\right)$. The balloon materials are either a "ceramic" (alumina, $E_{\mathrm{mon}}=382 \mathrm{GPa}$ ), a "glass ceramic" (silceram, $E_{\mathrm{mon}}=121 \mathrm{GPa}$ ), or a "metal" (mild steel, $E_{\text {mon }}=210 \mathrm{GPa}$ ), and the material constants further required by (3) and (4) are respectively $\{\beta=13.76$ and $c=0.46\},\{\beta=2.21$ and $c=0.35\}$, and $\{\beta=6.15$ and $c=0.38\}$. The ensuing Figure 1 shows the composite stiffness simulation outputs for all the above materials, when the spherical reinforcements are "compact spheres" (upper diagrams) or "hollow spheres" (lower diagrams) of the same size $(D=50 \mu \mathrm{m})$.

The stiffness trends displayed in the above plot by all microsphere-reinforced composites seem to be in general agreement with experimental observations, whereas the microballoon-reinforced (syntactic) composites trends show instead a severe stiffness decay as an increasing number of hollow spheres is added to the matrix material. 



FIGURE 2: Syntactic composites stiffness as predicted by this model (4) and Toda's model (6), when the same microballoons moduli $E_{b}$ are used in either model and $0 \leq V_{b} \leq 0.52$.

In order to further investigate this peculiar stiffness behaviour, predictions from the "modified model"/(4) were next compared with those obtained by a model (hereafter termed Toda's model [1]) derived from Bruggeman's rule of mixtures for particulate composites. Such model is consistent with experiments [13] for silica balloons reinforced epoxy composites and further described by

$$
E_{B}=E_{m}\left\{1+3 V_{b} \frac{\left(E_{b} / E_{m}\right)-1}{\left(E_{b} / E_{m}\right)+2}\right\} .
$$

For sake of a correct comparison, the same balloon moduli $E_{b}$ were adopted in both models for all simulated composites, in accordance with two alternative cell-porosity exponents $(k=2.7$ and $k=2.9)$. The outcome is plotted in Figure 2.

As shown above, either model defines similar stiffness decay trends and both are sensitive to cell-porosity exponents (i.e., larger porosity-exponents lead to smaller composites stiffness). Yet Toda's model anticipates larger stiffness decay rates than this model and predicts, unlike (4), enhanced decays even beyond the isotropy upper bound since the balloons volume fraction in (6) is free to range from "zero" to "one" without concentration restrictions.

As previously referred, a complementary feature of this modified model is the assessment of composites density ( $\rho_{S}$ and $\rho_{B}$, for sphere-reinforced and balloon-reinforced composites, resp.). These forecasts can be coupled to the composites stiffness (or any other mechanical property) in order to derive theoretical values of specific properties.

The theoretical density of microspheres-reinforced composites $\left(\rho_{S}\right)$ can be achieved by equating both the mass and volume of the cubic volume element: the spherical particle mass $\left\{(4 \pi / 3) R^{3} \times \rho_{\text {cer }}\right\}$; the surrounding matrix mass $\left\{\left[1-(4 \pi / 3) R^{3}\right] \times \rho_{m}\right\}$; the virtual particle size $\{R=$ $\left.0.62 V_{s}^{1 / 3}\right\}$; the cell volume $\{1 \times 1 \times 1\}$. This yields

$$
\begin{aligned}
\rho_{S} & =\left[\left(0.998 V_{s}\right) \rho_{\text {cer }}+\left(1-0.998 V_{s}\right) \rho_{m}\right] \\
& \sim\left[V_{s} \rho_{\text {cer }}+\left(1-V_{s}\right) \rho_{m}\right],
\end{aligned}
$$

where $\rho_{\text {cer }}$ is the ceramic material density and $V_{s}$ is the spherical particles volume fraction.

As for the microballoons-reinforced (syntactic) composites, a similar reasoning leads to

$$
\rho_{B}=\rho_{S}-f(n) \cdot \rho_{\text {cer }},
$$

where $\rho_{B}$ is the balloons composite overall density, and $f(n)=\left(N V_{O} / n\right)$ is a function of the number $n$ of fractured microballoons at a given stage of a composite loading test, $N$ is the total number of sound microballoons in the composite at the beginning of the test, and $V_{O}$ is the composite void volume fraction arising from pore formation within the matrix as $n$ microballoons become wide open upon fracture.

\section{Syntactic Composites-The "Effective" Variables}

Since the microstructures of most syntactic composites incorporate balloon-size distributions rather than single-size balloon dispersions, and the ceramic microballoons strength becomes adversely affected by large balloon diameters, the weakest microballoons become prone to fracture as the composites are subjected to increasing external loading (as in tensile or fracture toughness testing). Once fractured at a given applied stress level, these particles cease to act as "reinforcing units" and rather behave as nonreinforcing "inclusions", so they ought not to be taken into account in further reinforcement volume fraction evaluations. The fracture of reinforcing balloons within a loaded syntactic composite modifies this material anticipated performance, because composition-dependent mechanical properties may be impaired while some other properties may be damaged by the growing void volume fraction.

Since the fracture of microsphere-reinforced composites is mainly due to "matrix cracking" operating mechanisms, whereas the fracture of microballoon-reinforced composites is rather ascribed to extensive "particle cracking" [1], the concentration correction required by the surviving balloons will only aim at (4) for syntactic composites.

Such a correction implies to define an "effective reinforcement volume fraction" ( $\left.V_{\text {eff }}\right)$ which can be related to the nominal volume fraction $\left(V_{b}\right)$ prevailing at the stage where no applied forces existed, by means of a relationship involving the ratio of the estimated number of fractured balloons $(n)$ to the total population of original balloons $(N)$ such as

$$
V_{\text {eff }}=V_{b}\left(1-\frac{n}{N}\right)
$$

In the above, $n$ will be estimated in due course by fitting to its evolution an adequate statistical distribution (e.g., 


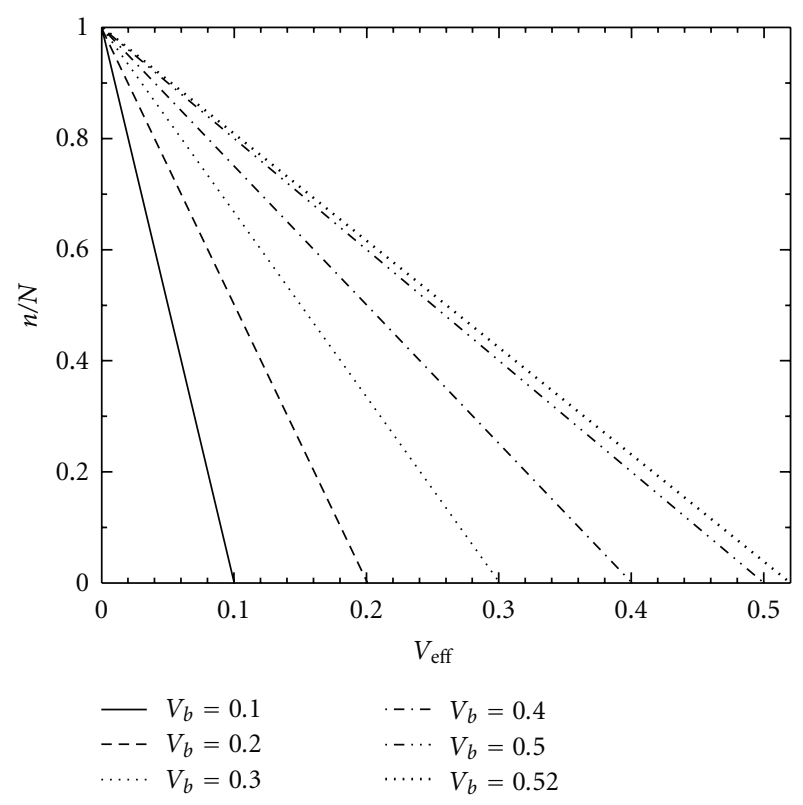

FIgURE 3: Linear dependence of $V_{\text {eff }}$ on the fraction $(n / N)$ of fractured balloons upon each composite specimen (tensile) loading.

Weibull distribution, for brittle solids) to assess the number of surviving balloons at each applied stress level. And $N$ is an experimental parameter related to the composites controlled manufacturing eventually made available from routine gravimetric analysis.

The correction (9) is an obvious generalization of the balloons original volume fraction, as $V_{\text {eff }} \equiv V_{b}$ when no reinforcing balloon has been destroyed yet $(n=0)$. At any other subsequent stage of balloons fracture, $0<(n / N)<1$ and therefore $V_{\text {eff }}<V_{b}$. This "effective" variable dependence on the $(n / N)$ ratio is illustrated by Figure 3 .

The reinforcement concentration correction, by means of which the nominal balloons volume fraction is replaced by an "effective" value, also implies a similar correction of the virtual particle radius $R=0.62 V_{b}^{1 / 3}$. This is so because the fewer reinforcing particles exist within the composite as external loading increases, the smaller must also be the virtual sphere size. Hence

$$
R_{\mathrm{eff}}=0.62 V_{\mathrm{eff}}^{1 / 3}=0.62\left[V_{b}\left(1-\frac{n}{N}\right)\right]^{1 / 3} .
$$

As before, this "effective" variable dependence on the $(n / N)$ ratio is depicted in Figure 4.

As both "effective" variables $V_{\text {eff }}$ and $R_{\text {eff }}$ are related in Figures 3 and 4 to the same $(n / N)$ variable, they can also be related to one another as illustrated in Figure 5.

The above diagram seemingly comprises three distinct particle growth regimes: a first high-curvature segment (up to $V_{\text {eff }} \sim 0.2$ ) is followed by a second low-curvature segment (up to $V_{\text {eff }} \sim 0.52$ ), where the particle growth proceeds at nearly constant rate, and beyond which a third (not shown) regime extends up to a maximum volume fraction.

The combined first and second stages spread over a concentration range, where a balloon's dispersion remains



Figure 4: Curvilinear dependence of $R_{\text {eff }}$ on the fraction $(n / N)$ of fractured balloons upon each composite specimen (tensile) loading.

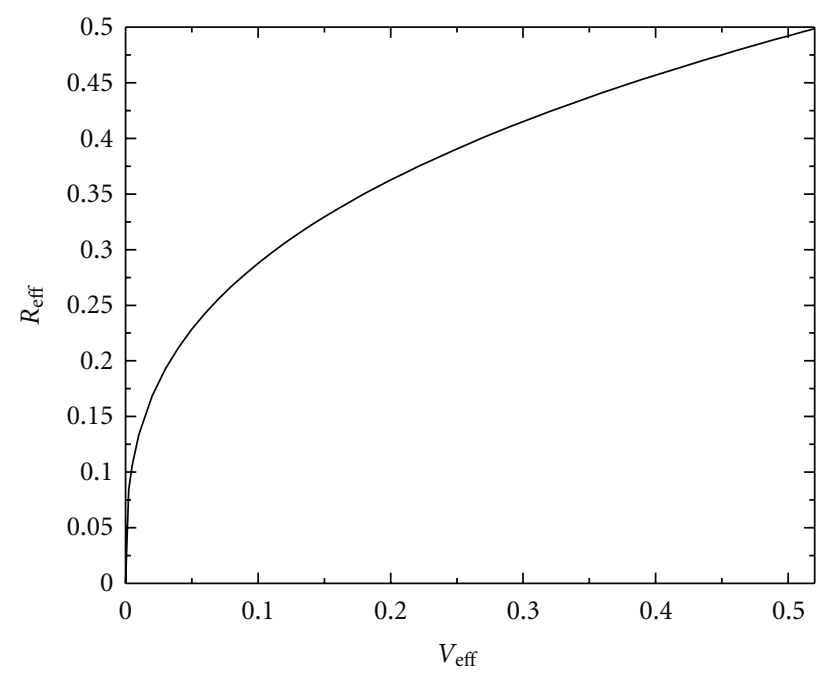

FIGURE 5: Virtual particle size, within the representative volume element, plotted versus intact balloons volume fraction.

isotropic, as certified by a simulation study [14], where the packing of uniform-sized spheres could reach a volume fraction range [0.5-0.6] without traces of lattice formation (in the form of preferential directions) inside the "particle" arrangements. Conversely, the third (not depicted) stage is likely to be an anisotropic domain as its [0.52] lower bound is the volume fraction for a "simple cubic lattice" extending throughout a syntactic composite.

Figures 3, 4, and 5 can be assembled all together so as to display the mutual relationships between the "effective" variables and the fractured (nonreinforcing) balloons ratio, leading to the following Figure 6 summary diagram. 


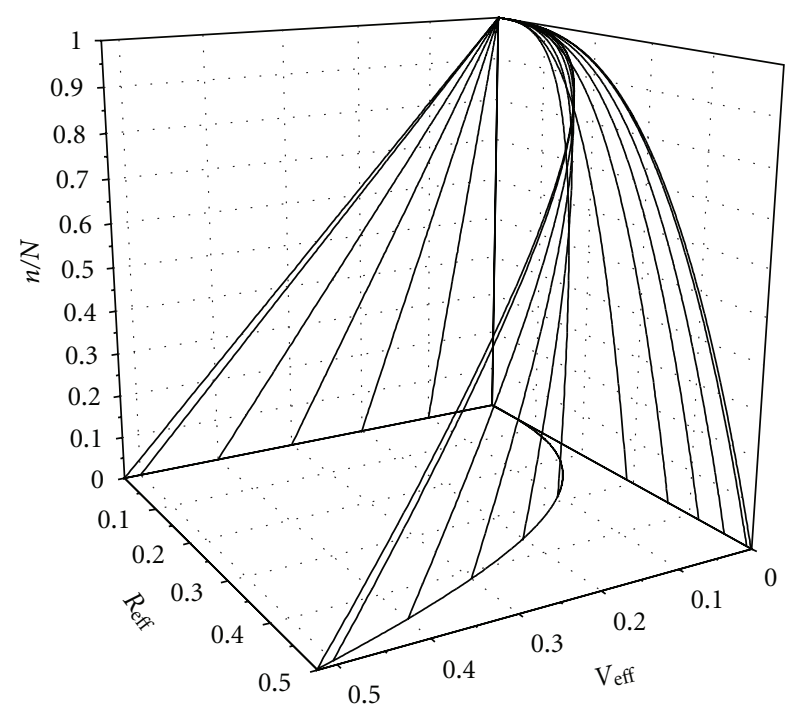

Figure 6: A compounded view of the "effective" variables and the unbound balloons ratio.

Following the "effective" variables definition, the formerly derived (4) for unloaded syntactic composites can now be rewritten for the loaded composites case, as below

$E_{B^{(n)}}=E_{m}\left\{\left(1-2 R_{\mathrm{eff}}\right)+\frac{2 c}{\sqrt{c-R_{\mathrm{eff}}^{2}}} \times \arctan \left(\frac{R_{\mathrm{eff}}}{\sqrt{c-R_{\mathrm{eff}}^{2}}}\right)\right\}^{-1}$,

where $R_{\text {eff }}=0.62\left[V_{b}\left(\left(N-n\left(\sigma_{b}\right)\right) / N\right)\right]^{1 / 3}$ is now a function of $n$, which becomes itself a function of the applied balloon stress $\sigma_{b}$, and the material constant $c=\left[\pi\left(1-\left(E_{b} / E_{m}\right)\right)\right]^{-1}$ is evaluated as before.

From the above, it is clear that (11) stiffness estimates become impossible unless a prior $n$ input is provided. This requirement can be met by a statistical distribution which will assess the number of surviving balloons $\left[N-n\left(\sigma_{b}\right)\right]$ at each applied stress level, to be further described.

\section{Syntactic Composites-The Statistical Distributions}

The ensuing treatment will resort to the Weibull statistics as such a distribution was formerly designed to suit the fracture of brittle solids (e.g., ceramic microballoons, and derived composites). The Weibull's approach aims at predicting brittle solids tensile strengths, based on (widely scattered) tensile strengths gathered from a batch of $\sim 20-30$ nominally identical composite samples. Once these experimental tensile strengths are arranged in order of increasing failure stresses and a failure stress probability is assigned to each failure by an adequate estimator [15], a relationship between "composite strength" and "composite failure probability" can be derived from a straight line regression fitting [16].

The sole relevant information for the present modelling analysis would, in principle, just be the tensile strength data provided by the composite specimens batch. Nevertheless, it may be referred for sake of completeness that a certain "probabilistic strength" can be assigned to the whole lot of brittle composites following after a $\left\{\ln \left[\ln \left(1 /\left(1-P_{f}\right)\right)\right]\right\}$ plot versus $\left\{\ln \boldsymbol{\sigma}_{B}\right\}$, where $P_{f}$ is the failure probability of each tensile specimen and $\sigma_{B}$ is the corresponding composite fracture $(\boldsymbol{\sigma})$ stress. The outlined procedure enables a graphical measurement of two "Weibull distribution" parameters: the Weibull modulus $m$ of tensile-tested samples material, and the scale factor $\sigma_{O}$. These two parameters are respectively identified with the slope and the vertical axis interception of the following statistical (oblique) straight line:

$$
\left\{\ln \left[\ln \frac{1}{1-P_{f}}\right]\right\}=m\left\{\ln \sigma_{B}\right\}-\left\{m \cdot \ln \sigma_{O}\right\}
$$

Once $m$ and $\sigma_{O}$ are assigned some numerical values, the probabilistic strength of a particular composites batch is then expressed by a "two-parameters" Weibull distribution

$$
\left\{1-P_{f}\right\}=\exp \left\{-\left(\frac{\sigma_{B}}{\sigma_{O}}\right)^{m}\right\}
$$

where $\left(1-P_{f}\right)$ is the fraction of identical composite samples which survive loading to a tensile stress $\sigma_{B}$ (In this notation, $\sigma_{B}$ is a continuous variable ranging from zero upwards, whereas $\sigma_{B}$ is a discrete variable).

The above mentioned composite specimens tensile strengths must now be related to the corresponding balloon applied tensile stresses. Should the tensile tests be conducted on a batch of continuous fibre composites, whose elastic fibres are loaded along their axes (as assumed by Voigt "constant strain" model) and whose matrices are also elastic, the stress acting on each fibre would be calculated from theoretical stress ratios as per $\sigma_{f}=\left(E_{f} / E_{c}\right) \sigma_{c}$, where subscripts " $f$ " and " $c$ " stand respectively for "fibre" and "composite" materials. A resembling situation occurs in unidirectional discontinuous short fibre composites, where a similar term remains as an "upper estimate" $\left(\sigma_{f}\right)_{\operatorname{MAX}}=$ $\left(E_{f} / E_{c}\right) \sigma_{c}$ of the fibre stress profile imposed on each of the parallel fibres array, while the composite itself is subjected to a tensile stress $\sigma_{c}$. As for syntactic composites tensile tests, a similar reasoning is to be adopted, such that any uniaxial "normal (tensile) stress" along a balloon equator may also be regarded as an "upper estimate" $\left(\sigma_{b}\right)_{\operatorname{MAX}}=\left(E_{b} / E_{B}\right) \sigma_{B}$ of the balloon applied tensile stresses. The mechanical validity of such an assumption becomes progressively more accurate as the balloons diametral cross-sections mimic more closely the short fibres transverse sections, as the balloons grow smaller and thicker. Even though the surface of an individual balloon may be simultaneously subjected to a variety of uniaxial tensile stresses, none of these is to be larger than the maximum interfacial normal stress applied along an equator under uniaxial tensile loading. Such a stress is thus the most plausible source of balloons cracking, so that the above $\left(\sigma_{b}\right)_{\text {MAX }}$ estimate will be regarded in this analysis as the balloon loading $\sigma_{b}$ held responsible for balloons suppression within tensile loaded syntactic composites

$$
\sigma_{b}=\left(\frac{E_{b}}{E_{B}}\right) \sigma_{B}
$$


In the above, $E_{b}$ is the balloon modulus as per (5), $E_{B}$ is the unloaded syntactic composite stiffness as per (4), and $\sigma_{B}$ is any of the monitored experimental uniaxial tensile stresses applied to the syntactic composite during the specimen tensile test. As $\sigma_{B}$ is progressively increased during the testing, $\sigma_{b}$ is proportionally increased as well, due to the growing strength of the remaining stronger balloons.

The ceramic balloons are brittle solids, so their strength must comply with a two-parameters Weibull distribution similar to that described by (13). Hence

$$
\left\{1-\frac{n}{N+1}\right\}=\exp \left\{-\left(\frac{\sigma_{b}}{\widehat{\sigma}_{0}}\right)^{\hat{\mathrm{m}}}\right\},
$$

where $n /(N+1)$ is the statistical estimate [15] of the balloons probability of failure at $\sigma_{b}$ stress; $\hat{\mathbf{m}}$ and $\hat{\sigma}_{0}$ are the balloons Weibull distribution constants. These constants are obtained by applying (12) to the balloons strengths, which were estimated from the composites experimental fracture stresses by means of (14). And $N$ is an experimental constant related to the composites nominal volume fraction $V_{b}$.

Since $N$ is a huge number (so that $N+1 \sim N$ ), and ( $1-$ $n / N)=\left(V_{\text {eff }} / V_{b}\right)$, the above equation then yields

$$
\sigma_{b}=\hat{\sigma}_{0} \cdot \sqrt[\hat{\mathrm{m}}]{\ln \left(\frac{V_{b}}{V_{\text {eff }}}\right)}
$$

a central relationship whose parametric variables are all assigned concrete values at this stage but $V_{\text {eff }}$, as the required $n$ input has not been established yet.

\section{Syntactic Composites-The Stiffness Statistical Model}

The statistical model for stiffness of loaded syntactic composites arises from $E_{B}(n)$ evaluations as per (11), coupled to the $n$ input values as defined by the (14) and (16) simultaneous system. If this system is solved for the experimental uniaxial tensile stress $\sigma_{B}$ applied to each syntactic composite specimen, the following relationship is derived:

$$
\sigma_{B}=\left\{\left(\frac{E_{B}}{E_{b}}\right) \cdot \hat{\sigma}_{0} \cdot \sqrt[\hat{\mathrm{m}}]{\ln \left(\frac{V_{b}}{V_{\text {eff }}}\right)}\right\}
$$

and the missing $n$ input becomes then established (through $V_{\text {eff }}$ values) at any stage of the tensile test.

Equation (17) can be read as per the "straight direction" if $\sigma_{B}$ is either arbitrary or experimental, in order a compatible $n$ value to be obtained. But this equation can also be read as per the "reverse direction", to the purpose of estimating the tensile stress required for fracturing an arbitrary number $n$ of reinforcing balloons. This particular simulation may be an useful tool for damage assessment of fabricated composites (e.g., by establishing quality control charts). Combined tables of experimental tensile stress $\sigma_{B}$ coupled to "loaded" syntactic composites stiffness $E_{B}(n)$ predictions (as per (11)) can also be built up, aiming at checking whether a common linear-elastic relationship may exist between them-in which case a composite proportional limit elongation can further be set up.
Equation (17) also satisfies an "initial" boundary condition at a stage where no balloon has collapsed (if $n=0$ then $V_{\text {eff }} \equiv V_{b}$, so $\left.\sigma_{B}=0\right)$; yet the "final" boundary condition at a stage where a massive number of balloons has failed (if $n \rightarrow N$ then $V_{\text {eff }} \rightarrow 0$, so that $\left|\sigma_{B}\right| \rightarrow \infty$ ) is only approached asymptotically, meaning that full destruction of all reinforcing microballoons in the composite can never be achieved for all practical purposes because $(0 \leq) n<N$.

\section{Conclusions}

A theoretical analysis has been conducted on the elastic stiffness of spherical-particles reinforced composites. Composites were assumed to have thin particle-matrix interfaces; both particle and matrix constituents were further supposed to have identical Poisson's ratios and to be subjected to the same strain upon composite loading. While spherical (either compact or hollow) reinforcements were not significantly cracked by external tensile stresses, the composites predicted stiffness was simply derived from the states of macroscopic stress and strain within a representative unit volume element. This was not the loaded syntactic composites case, whose stiffness predictions might otherwise be affected by severe microballoons destruction due to moderate-to-high applied tensile stresses. A complementary approach was therefore devised for this specific case, which involved the definition of some parametric variables related to the number of surviving balloons at a given stress level, as well as assigning brittle solids strength statistics to ceramic microballoons and their syntactic composite materials. The overall output of the above contributions is a statistical stiffness model for microballoons-reinforced tensile loaded composites. This model reliability can seemingly be confirmed from theoretical predictions (e.g., (7) forecasts) anticipated by established models and composite materials fundamentals and also from the agreement (in Figure 2) between experimental predictions made by two utterly distinct approaches (this model (4)/Toda's model (6)) before a given set of experimental results [13]. It is nevertheless worthy to compare this model foundations against those from other classical analytical models [5-9] already quoted in this paper.

The two independent elastic constants of an isotropic random ensemble of elastic spherical inclusions embedded in a continuous, isotropic, and elastic matrix can be provided by numerical (finite element) methods as well as by three current analytical models: the Mori-Tanaka mean-field analysis [5] (further extended to isotropic dispersions by Benveniste [6]); the Christensen and Lo generalized self-consistent method [7]; and Torquato third-order approximation [8]. Yet numerical solutions can only be obtained if a composite representative volume element is first of all established so as the enclosed particles dispersion to be statistically isotropic (thereby rendering all directions equivalent inside that volume), and the cubic cell is last assigned some periodic boundary conditions before computing the volume element elastic constants. As for $[5,6]$, this approximation requires the stress and strain fields in the matrix and in the inclusion to be adequately represented by their volume-average values, which are to account for the elastic interactions 
between the inclusions. The analysis [7] assumes a matrixcoated spherical inclusion to be embedded into an effective medium, in such a way the whole ensemble behaves as a three-phase composite whose properties are related to a differential equations set. The composite elastic constants can then be obtained by integrating the differential equations set for appropriate boundary conditions. Finally, the approach [8] resorts to an exact series of expansion for the effective stiffness tensor, which is truncated after third-order terms while the first series terms are explicitly given as a function of two statistical correlation parameters (whose values can be found in [9] for a three-dimensional random distribution of identical, impenetrable hard spheres as a function of the sphere volume fraction). These analytical models also require some additional information on the upper and lower bounds for the elastic properties of isotropic, twophase composites. Such information is currently gathered by variational principles, which may certify how the inclusions are distributed throughout the matrix.

Much like the previous analytical models, this micromechanics model $[3,4]$ also assumes some of the physical approximations described above: strain fields in the matrix and in the inclusion are to be identical as well as the respective Poisson's ratios (a best suited condition to metal/ceramic combination); and both the stress and strain fields imposed on a composite by external stresses can be reproduced in a representative cubic unit cell. Unlike the above models, however, this micromechanics model includes two additional distinctive features: a mandatory balloons volume fraction replacement by an "effective" value (which only takes into account the sound balloons prevailing up to a given composite stress level) and the Weibull statistics incorporation into this model, so as to account for the balloons surface defects on the composites ultimate mechanical properties. All these separate contributions merge into this analytical model so as to make more obvious its main characteristics: it is handy, it allows easy modelling of reinforcement morphologies, it embodies a self-evaluation criterion for composite (an)isotropy, and it only requires the same tensile tests methodology that is used by the Weibull's approach to assess brittle solids tensile strengths.

Designing with syntactic composites depends on these materials performance when tested for a particular property, while balloons enhanced annihilation is imposed by growing applied loads. The "elastic stiffness" property behaviour, for example, is described in Figure 2-where its rise becomes noticeable as the balloons effective volume fraction is decreased. Such trend is sustained up to a limit value compatible with the balloons full destruction. As for the composite "mechanical strength," an opposite trend may be expected, since the progressive balloons annihilation scales down the available sites where matrix/reinforcement loadtransfer processes might occur. Loaded syntactic composites strength is thus expected to fall, as the (sound) balloons effective volume fraction is reduced. A similar reasoning holds for the "fracture toughness" of loaded syntactic composites, because the growing number of minute cavities within the matrix provides numerous sites for cracks nucleation, whose subsequent propagation ultimately leads to composite fracture. Syntactic composites fracture toughness is therefore likely to decrease as the reinforcing balloons volume fraction is also reduced.

As a final remark, all the engineering properties cited above (and stiffness in particular) are to behave as "variable parameters" for as long a period as the loading test duration. Hence, such variability also applies to any other elastic constant eventually bound to stiffness by any conceivable fundamental mechanical relationship, providing it is valid for isotropic composites.

\section{References}

[1] H. Toda, "Porous particle composites," in Metal and Ceramic Matrix Composites, B. Cantor, F. Dunne, and I. Stone, Eds., vol. 20, pp. 350-366, Institute of Physics Publishing, London, UK, 2004.

[2] B. Paul, "Prediction of elastic constants of multiphase materials," Transactions of the Metallurgical Society of AIME, vol. 218, pp. 36-41, 1960.

[3] J. D. Botas, A. Velhinho, and R. J. C. Silva, "Elastic behaviour of spherical particles reinforced metal-matrix composites," Materials Science Forum, vol. 587-588, pp. 202-206, 2008.

[4] J. D. Botas, A. Velhinho, and R. J. C. Silva, "A theoretical approach to the elastic behaviour of compact and hollow spherical particles reinforced metal-matrix composites," International Journal of Materials Research, vol. 101, no. 6, pp. 752757, 2010.

[5] T. Mori and K. Tanaka, "Average stress in matrix and average elastic energy of materials with misfitting inclusions," Acta Metallurgica et Materialia, vol. 21, no. 5, pp. 571-574, 1973.

[6] Y. Benveniste, "A new approach to the application of MoriTanaka's theory in composite materials," Mechanics of Materials, vol. 6, no. 2, pp. 147-157, 1987.

[7] R. M. Christensen and K. H. Lo, "Solutions for effective shear properties in three phase sphere and cylinder models," Journal of the Mechanics and Physics of Solids, vol. 27, no. 4, pp. 315330, 1979.

[8] S. Torquato, "Effective stiffness tensor of composite media: II. Applications to isotropic dispersions," Journal of the Mechanics and Physics of Solids, vol. 46, no. 8, pp. 1411-1440, 1998.

[9] S. Torquato, "Random heterogeneous media: microstructure and improved bounds on effective properties," Applied Mechanics Reviews, vol. 44, no. 2, pp. 37-76, 1991.

[10] W. J. Drugan and J. R. Willis, "A micromechanics-based nonlocal constitutive equation and estimates of representative volume element size for elastic composites," Journal of the Mechanics and Physics of Solids, vol. 44, no. 4, pp. 497-524, 1996.

[11] W. J. Drugan, "Micromechanics-based variational estimates for a higher-order nonlocal constitutive equation and optimal choice of effective moduli for elastic composites," Journal of the Mechanics and Physics of Solids, vol. 48, no. 6, pp. 1359-1387, 2000.

[12] M. Hartmann, I. Crossmann, K. Reindel, and R. F. Singer, "Behaviour of composite structures," in Metal Foams and Porous Metal Structures, J. Banhart, M. F. Ashby, and N. A. Fleck, Eds., pp. 331-336, Metall Innovation Technologie (MIT), Bremen, Germany, 1999.

[13] H. Toda, H. Kagajo, K. Hosoi et al., "Evaluation of mechanical properties of hollow particle reinforced composites and 
analyses aimed at their improvement," Journal of the Society of Materials Science, Japan, vol. 50, no. 5, pp. 474-481, 2001.

[14] J. Segurado and J. Llorca, "A numerical approximation to the elastic properties of sphere-reinforced composites," Journal of the Mechanics and Physics of Solids, vol. 50, no. 10, pp. 21072121, 2002.

[15] A. C. Fischer-Cripps, Introduction to Contact Mechanics, Springer, New York, NY, USA, 2000.

[16] A. R. Bunsell and J. Renard, "Fundamentals of fibre reinforced composite materials," in Series in Materials Science and Engineering, B. Cantor, M. J. Goringe, and E. Ma, Eds., pp. 6671, Institute of Physics Publishing, London, UK, 2005. 



The Scientific World Journal

Submit your manuscripts at

http://www.hindawi.com

\section{World Journal}

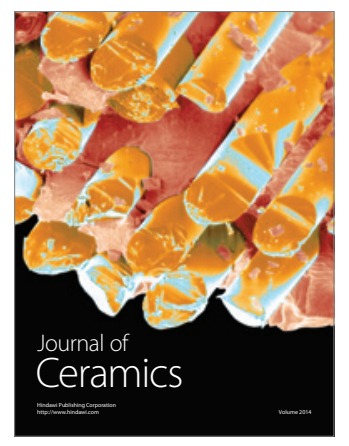

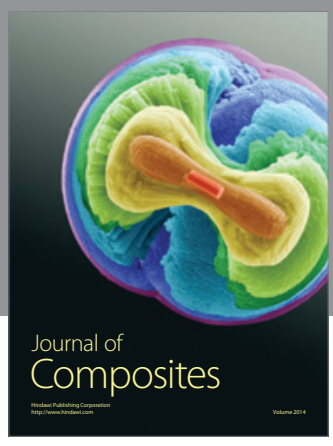
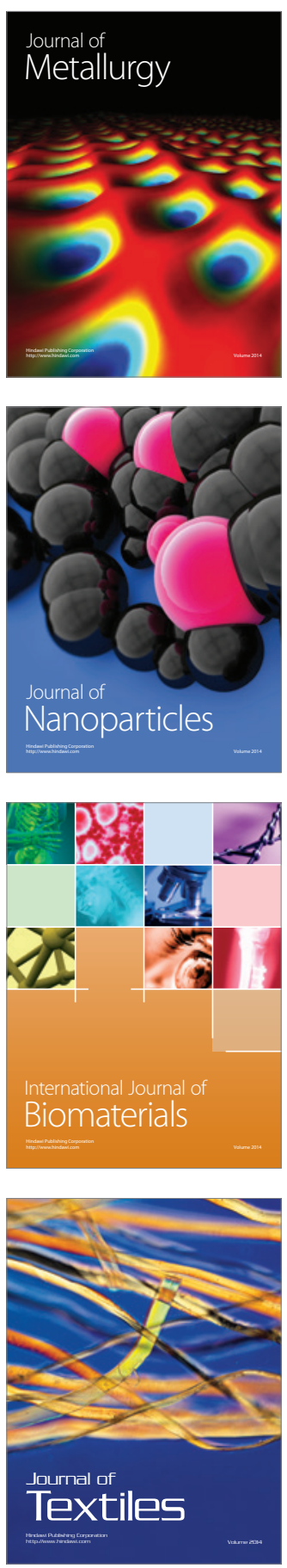